\title{
COMPARAÇÃO DA QUALIDADE SANITÁRIA ENTRE AMOSTRAS DE Peumus boldus Molina INDUSTRIALIZADAS E ARTESANAIS DO MUNICÍPIO DE CURRAIS NOVOS, RN
}

\author{
F. G. M. Medeiros ${ }^{1}$, F. A. G. Rocha ${ }^{2}$, U. K. L. Medeiros3, L. I. S. Dantas ${ }^{4}$, L. O. Araújo ${ }^{5}$ e M. F. F. Araújo ${ }^{6}$ \\ $1,2,3,4,5$ Instituto Federal do Rio Grande do Norte e ${ }^{6}$ Universidade Federal do Rio Grande do Norte \\ angelo.gurgel@cefetrn.br1
}

Artigo submetido em março/2012 e aceito em junho/2012

\section{RESUMO}

As plantas medicinais, embora constituam válida vertente da Medicina Tradicional, quando mal utilizadas representam risco potencial a saúde humana. O consumo de produtos contaminados pode gerar intoxicações diversas por subprodutos advindos de microrganismos patogênicos. $O$ presente trabalho objetivou a avaliação da qualidade microbiológica de amostras de Peumus boldus Molina artesanais, comercializadas na feira popular, e compará-la com a qualidade microbiológica de amostras da mesma espécie industrializadas, comercializadas no comércio varejista local. Foram quantificados: aeróbios mesófilos (ágar Padrão de Contagem, $35 \pm 1^{\circ} \mathrm{C} / 24 \mathrm{~h}$ ), coliformes totais/Escherichia coli (teste presuntivo: caldo LST, $35 \pm 0,5^{\circ} \mathrm{C} / 24-48 \pm 2$; $\mathrm{CT}$ : caldo $\mathrm{VB}, 35 \pm 0,5^{\circ} \mathrm{C} / 24-48 \pm 2$; $\mathrm{E}$. coli: caldo $\mathrm{EC}, 44,5 \pm 0,2^{\circ} \mathrm{C} / 24 \pm 2 \mathrm{~h}$; ágar L-EMB, $35 \pm 0,5^{\circ} \mathrm{C} / 24 \pm 2 \mathrm{~h}$ ), bolores e leveduras (ágar Batata Dextrosado Acidificado, $25 \pm 1^{\circ} \mathrm{C} / 5$ dias) e Staphylococcus aureus (ágar Baird-Parker, $35-37^{\circ} \mathrm{C} / 24 \pm 2 \mathrm{~h}$ ). Para a confirmação de $\mathrm{E}$. coli, colônias foram submetidas às provas bioquímicas da série IMViC. A E. coli estava presente em $10 \%$ das amostras, o S. aureus em 50\%, bolores e leveduras em $80 \%$ e aeróbios mesófilos em $100 \%$ das amostras analisadas. O estudo revelou que as amostras industrializadas e artesanais não apresentaram as recomendações de padrões sanitários e microbiológicos adequados para o comércio, caracterizando-se como riscos potenciais à Saúde Pública.

PALAVRAS-CHAVE: plantas medicinais, medicina tradicional, comércio varejista, microrganismos patogênicos.

\section{SANITARY QUALITY COMPARISSON BETWEEN SAMPLES OF INDUSTRIALIZED AND HANDMADE Peumus boldus Molina IN CURRAIS NOVOS - RN}

\begin{abstract}
Medicinal plants, though they constitute a valid slope of Traditional Medicine, can represent a potential risk to human health when misused. The consumption of contaminated products may cause severe poisoning by byproducts that occur in pathogenic microorganisms. This paper aims to evaluate the micro biological quality of handmade Peumus boldus Molina samples, commercialized at the popular market and compare them in terms of micro biological quality with the same industrialized types, dealt at the local retailing market. The following ones were specified: aerobic mesophilic (plate count agar, $35 \pm 1^{\circ} \mathrm{C} / 24 \mathrm{~h}$ ), total coliforms/ Escherichia coli ( Presumptive test: LST broth,
\end{abstract}

$35 \pm 0,5^{\circ} \mathrm{C} / 24-48 \pm 2 ; \mathrm{CT}: \mathrm{VB}$ broth, $35 \pm 0,5^{\circ} \mathrm{C} / 24-48 \pm 2$; $\mathrm{E}$. coli: EC broth, $44,5 \pm 0,2^{\circ} \mathrm{C} / 24 \pm 2 \mathrm{~h}$; L-EMB agar, $35 \pm 0,5^{\circ} \mathrm{C} / 24 \pm 2 \mathrm{~h}$ ), molds and yeasts ( potato dextrose agar, $25 \pm 1^{\circ} \mathrm{C} / 5$ days) and Staphylococcus aureus (BairdParker agar, $35-37^{\circ} \mathrm{C} / 24 \pm 2 \mathrm{~h}$ ). For confirming the E. coli, colonies were put to biochemical proofs of the series IMViC. The E. coli was present in $10 \%$ of the samples, the S. aureus in $50 \%$, molds and yeasts in $80 \%$ and aerobic mesophilic in $100 \%$ of the analized samples. The study found out that neither the industrialized samples nor the handmade ones were according to the sanitary and microbiological standard specifications for the market, resulting in potential risks for public health.

KEY-WORDS: medicinal plants, traditional medicine, retailing market, pathogenic microorganisms. 


\section{COMPARAÇÃO DA QUALIDADE SANITÁRIA ENTRE AMOSTRAS DE Peumus boldus Molina INDUSTRIALIZADAS E ARTESANAIS DO MUNICÍPIO DE CURRAIS NOVOS, RN}

\section{INTRODUÇÃO}

A Medicina Tradicional baseia-se em conhecimentos milenares passados através das gerações, contemplando dentre outros recursos, o uso de espécies vegetais com finalidade terapêutica, as plantas medicinais. A ampla aceitação destas, em muitos casos com ações biológicas comprovadas estimulam o consumo e, consequentemente, o comércio formal e informal. Entretanto, a comercialização e o uso inadequados podem vir a representar riscos à saúde humana, tendo em vista a possível contaminação por microrganismos patogênicos. Dentre os contaminantes de natureza microbiana potencialmente presentes, destacam-se os fungos, a Escherichia coli e o Staphylococcus aureus, organismos produtores de toxinas termoestáveis.

O presente estudo objetivou analisar a qualidade microbiológica de amostras de Peumus boldus Molina coletadas no comércio informal (feira livre) e formal (supermercados) do município de Currais Novos/RN. Os resultados obtidos foram comparados aos níveis recomendados pela Organização Mundial da Saúde.

\section{FUNDAMENTAÇÃO TEÓRICA}

A cultura do uso de plantas medicinais no tratamento e na cura de enfermidades é tão antiga quanto a espécie humana (MACIEL et al., 2002). Nas regiões menos desenvolvidas, tais práticas assumem um papel de maior relevância, sendo muitas vezes a única alternativa de tratamento disponível aos usuários locais. Tal fato deve-se às dificuldades de acesso destas populações à medicina moderna e, por consequência, aos medicamentos alopáticos (NUNES et al., 2003).

A Medicina Tradicional e seus recursos resultam do acúmulo de conhecimentos advindos das interações entre as comunidades humanas e seu meio ambiente. Antecedendo em milênios a Medicina Alopática, a Medicina Tradicional é definida pela Organização Mundial da Saúde (OMS) como sendo a soma de todos os conhecimentos teóricos e práticos, explicáveis ou não, utilizados para obter-se o diagnóstico, prevenção e tratamentos físicos, mentais ou sociais (WORLD HEALTH ORGANIZATION, 1998).

As influências culturais aliadas à ampla aceitação das plantas medicinais pela população aumentam tanto o comércio formal, quanto o informal. Este último traz consigo a falta de fiscalização efetiva e atenção reduzida com a qualidade dos produtos e serviços ofertados aos consumidores. O comércio formal por sua vez, alimentado pela grande procura da população por artigos ditos "naturais", oferece produtos beneficiados, em geral na forma de chás prontos, em pó-solúvel ou ainda na forma de sachês, contendo partes de órgãos vegetais dessecados. Estes últimos geralmente destinam-se ao preparo de chás, em geral infusões, preparadas com a adição de água fervente sobre os sachês (ROCHA et al., 2010; VULCANO et al., 2008).

Não obstante a necessidade do comércio, seja esse formal ou não, de oferecer produtos comprovadamente seguros e eficazes (NUNES et al., 2003), estudos revelaram que a qualidade microbiológica apresentada por tais produtos vegetais não é a mais adequada: dentre outros problemas, falhas no controle de qualidade da matéria-prima e insumos ao longo da cadeia 
produtiva podem permitir a veiculação de uma microbiota, por vezes patogênica (BUGNO et al., 2006). Dentre os microrganismos de importância médico-sanitária potencialmente presentes nas plantas medicinais disponíveis à população, sejam elas industrializadas ou não estão as bactérias aeróbias mesófilas, a E. coli, o S. aureus e os fungos produtores de micotoxinas. Tais microrganismos quando presentes em níveis não recomendados em plantas medicinais utilizadas no preparo de chás, podem gerar agravos à saúde dos consumidores que as utilizem (BUGNO et al., 2006; ROCHA \& MEDEIROS, 2009; WORLD HEALTH ORGANIZATION, 1998; WORLD HEALTH ORGANIZATION, 2007).

O presente trabalho objetivou analisar a qualidade microbiológica de amostras de Peumus boldus Molina coletadas no comércio informal (feira livre) e formal (supermercados) do município de Currais Novos/RN, comparando os resultados obtidos com os parâmetros recomendados pela Organização Mundial da Saúde para material de origem vegetal destinado ao preparo de chás.

\section{MATERIAIS E MÉTODOS}

\subsection{Caracterização da Área Estudada}

As amostras foram coletadas na feira popular e no comércio varejista do município de Currais Novos (figuras 1 e 2), situado na mesorregião Central Potiguar e na microrregião Seridó Oriental, sob as coordenadas 6 $6^{\circ} 15^{\prime} 39,6^{\prime \prime}$ Sul, $36^{\circ} 30^{\prime} 54^{\prime \prime}$ Oeste, Estado do Rio Grande do Norte (BRASIL, 2005).

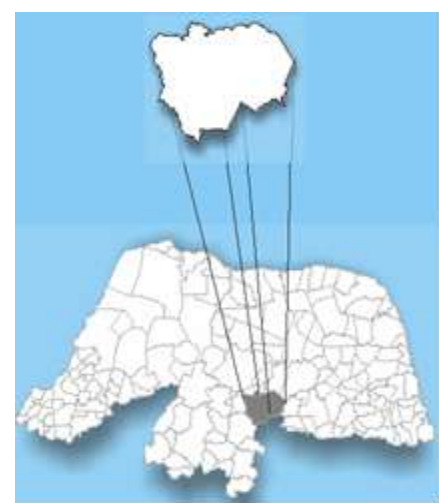

Figura 1: localização geográfica do município de Currais Novos, RN (BRASIL, 2005).

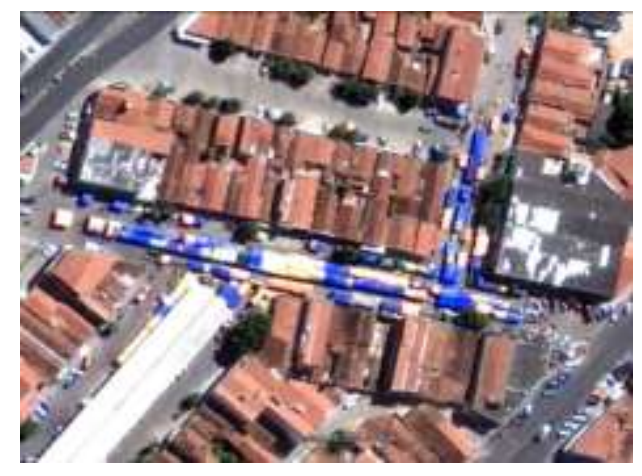

Figura 2: foto de satélite da área das feiras permanente e livre, bairro centro, município de Currais Novos, RN. Fonte: Google Earth ${ }^{\mathrm{m}}$

\subsection{Espécie Analisada e Procedimento de Coleta das Amostras}

Objetivando a reprodução dos procedimentos envolvidos na comercialização popular das plantas medicinais, as amostras foram embaladas pelos próprios comerciantes, empregando-se os materiais usualmente utilizados: jornais e sacos plásticos, no caso da feira popular, e sacolas plásticas, no caso do comércio varejista.

Nos locais descritos, foi coletado um total de dez amostras de P. boldus Molina (chá-de-boldo ou boldo-do-Chile), assim subdividido: na feira popular, foram coletadas quatro amostras artesanais constituídas por folhas dessecadas, inteiras e fragmentadas. No caso das amostras industrializadas, foram 
coletadas no mercado varejista três amostras de marcas distintas (em duplicata), totalizando seis amostras. As amostras eram constituídas por saches contendo folhas dessecadas e fragmentadas, acondicionadas em caixas de papelão lacradas por sacos plásticos invioláveis. O peso total de cada caixa era variável e sempre superior a 60 gramas. Os pacotes contendo as amostras foram acondicionados em sacos plásticos herméticos individuais (ziplock), objetivando minimizar contaminações posteriores. 0 material foi encaminhado ao Laboratório de Microbiologia de Alimentos/Biologia Molecular do IFRN/Câmpus Currais Novos, onde foi analisado, respeitando-se um lapso temporal máximo de duas horas entre a coleta e a análise.

\subsection{Preparo das Amostras e Diluições Seriais}

Alíquotas de $25 \mathrm{~g}$ de cada amostra foram individualmente adicionados a $225 \mathrm{~mL}$ de solução salina peptonada estéril, homogeneizando-se por agitação durante dois minutos. A partir desta diluição inicial (10-1), foram realizadas diluições decimais seriadas, também homogeneizadas, até 10-3. Quando necessário, foram realizadas diluições decimais seriadas até 10-6. Os procedimentos adotados nas análises microbiológicas seguiram os parâmetros e passos metodológicos descritos por Silva et al (2007).

\subsection{Quantificação de Bactérias Aeróbias Mesófilas, Bolores e Leveduras e S. aureus}

Utilizando-se a Contagem Direta em Placa com semeadura em superfície (spread plate), a alíquota de 0,1 $\mathrm{mL}$ de cada uma das diluições foi individualmente semeada em duplicatas de placas de petri, conforme a descrição: para aeróbios mesófilos, ágar padrão de contagem, com incubação em posição invertida a $35 \pm 10 \mathrm{C}$ por $24 \mathrm{~h}$; para quantificação de bolores e leveduras, ágar batata dextrosado acidificado, com placas incubadas em posição normal a $25 \pm 10 \mathrm{C}$ por cinco dias; para o $\mathrm{S}$. aureus, ágar Baird-Parker (ABP) suplementado com emulsão de gema de ovo em solução salina (1:1 v/v) e telurito de potássio $1 \%$, sendo as placas incubadas em posição invertida a $35 \pm 10 \mathrm{C}$ por $24 \mathrm{~h}$. Em todos os casos, após a incubação, as colônias (típicas no caso do $\mathrm{S}$. aureus) foram contadas e os resultados expressos em Unidades Formadoras de Colônia por grama - UFC/g.

\subsection{Contagem de Coliformes Totais/E. coli.}

Conforme Silva et al. (2007) foi utilizado o Método do Número Mais Provável (NMP). Um mL de cada diluição foi inoculado em triplicata, em tubos de ensaio contendo cada $10 \mathrm{~mL}$ de Caldo LST e tubo Duhran invertido. O período de incubação foi de $24 / 48 \pm 2 \mathrm{~h}$ a $35 \pm 0,5$ oC. A partir dos tubos com produção de gás foram transferidas alçadas para tubos de ensaio associados a tubos Duhran, contendo $10 \mathrm{~mL}$ de Caldo Verde Brilhante-Bile 2\% (VB) para análise de Coliformes Totais e $10 \mathrm{~mL}$ de Caldo EC para a análise de Coliformes Termotolerantes/E. coli. Os Tubos VB foram incubados em estufa a $35 \pm 0,50 \mathrm{C}$ por $24-48 \pm 2 \mathrm{~h}$ e os EC a $44,5 \pm 0,20 C$ por $24 \pm 2 \mathrm{~h}$ em banho-maria. A produção de gás nos tubos VB foi considerada positiva para coliformes totais. A partir dos tubos de EC com produção de gás foram retiradas alçadas e estriadas placas de petri contendo cerca de $15 \mathrm{~mL}$ de Agar Levine Eosina Azul de Metileno (L-EMB). As placas foram incubadas em posição invertida a $35 \pm 0,5 \circ \mathrm{C}$ por $24 \pm 2 \mathrm{~h}$. Três colônias típicas de cada placa foram inoculadas para as provas bioquímicas de Indol, VM, VP e Citrato (IMViC) e motilidade em meio SIM. Foram consideradas positivas as colônias com perfil + + - + + (biotipo 1) ou - + - + (Biotipo 2). Em ambos os casos, os resultados foram expressos em Número Mais Provável por grama (NMP/g).

\section{ANÁLISE E INTERPRETAÇÃO DOS DADOS}

Na Tabela 1, estão descritos os resultados das análises microbiológicas das dez amostras de Peumus boldus Molina.

Tabela 1 - Resultados das análises microbiológicas das amostras de Peumus boldus Molina ( P1 P4: produtores; $A-J$ : amostras). 


\begin{tabular}{|c|c|c|c|c|c|c|c|c|}
\hline \multicolumn{3}{|c|}{ Produtor/Amostra } & \multirow{2}{*}{$\begin{array}{l}\text { Planta } \\
\text { P. boldus }\end{array}$} & \multirow{2}{*}{$\begin{array}{c}\text { C. Totais } \\
\text { (NMP/g) } \\
<3,0\end{array}$} & \multirow{2}{*}{$\begin{array}{c}\begin{array}{c}\text { E. coli } \\
\text { (NMP/g) }\end{array} \\
\text { Ausente }\end{array}$} & \multirow{2}{*}{$\begin{array}{c}\begin{array}{c}\text { S. aureus } \\
\text { (UFC/g) }\end{array} \\
\text { Ausente }\end{array}$} & \multirow{2}{*}{$\begin{array}{c}\text { Bol. e Lev. } \\
\text { (UFC/g) }\end{array}$} & \multirow{2}{*}{$\begin{array}{c}\begin{array}{c}\text { Aer. Mes. } \\
\text { (UFC/g) }\end{array} \\
4,3 \times 10^{2}\end{array}$} \\
\hline \multirow{4}{*}{$\frac{\pi}{4}$} & \multirow{4}{*}{ P1 } & A. & & & & & & \\
\hline & & B. & P. boldus & $<3,0$ & Ausente & Ausente & $5,0 \times 10^{1}$ & $2,0 \times 10^{2}$ \\
\hline & & C. & P. boldus & 9,2 & 3,6 & $>2,8 \times 10^{2}$ & $3,3 \times 10^{2}$ & $3,8 \times 10^{3}$ \\
\hline & & D. & P. boldus & $<3,0$ & Ausente & $4,0 \times 10^{1}$ & $5,5 \times 10^{2}$ & $4,0 \times 10^{3}$ \\
\hline \multirow{6}{*}{ 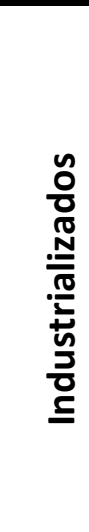 } & \multirow{2}{*}{ P2 } & E. & P. boldus & $<3,0$ & Ausente & $3,0 \times 10^{2}$ & $5,0 \times 10^{3}$ & $5,2 \times 10^{4}$ \\
\hline & & F. & P. boldus & $<3,0$ & Ausente & Ausente & $7,4 \times 10^{4}$ & $7,0 \times 10^{2}$ \\
\hline & \multirow{2}{*}{ P3 } & G. & P. boldus & 93 & Ausente & $1,5 \times 10^{2}$ & $7,3 \times 10^{5}$ & $1,65 \times 10^{4}$ \\
\hline & & $\mathrm{H}$. & P. boldus & $<3,0$ & Ausente & $2,0 \times 10^{2}$ & Ausente & $7,0 \times 10^{2}$ \\
\hline & \multirow[t]{2}{*}{ P4 } & I. & P. boldus & $<3,0$ & Ausente & Ausente & $1,0 \times 10^{4}$ & $5,0 \times 10^{3}$ \\
\hline & & J. & P. boldus & $<3,0$ & Ausente & Ausente & Ausente & $5,5 \times 10^{2}$ \\
\hline
\end{tabular}

Os resultados das análises microbiológicas mostram que em $100 \%$ das amostras industrializadas, a E.coli estava ausente. Já entre as amostras artesanais (de " $A$ " a " $D$ "), O microrganismo foi detectado em 25\%. A sua detecção é significativa por tratar-se de um bioindicador de contaminação fecal. Possivelmente veiculado no material através do solo, da água utilizada para a irrigação ou, até mesmo, pela manipulação inadequada do produto nos locais de produção e comercialização, sua deteç̧ão levanta a possível presença de parasitas e patógenos veiculados por fezes (FRANCO \& LANDGRAF, 2008).

O S. aureus foi verificado na mesma proporção, tanto nas amostras artesanais quanto nas industrializadas: $50 \%$. A sua deteç̧ão demonstra a possível exposição do material à manipulação inadequada em uma ou mais fases de sua cadeia produtiva, mesmo no caso dos produtos industrializados. A OMS não estabelece ou recomenda limites toleráveis para esta espécie. Contudo, por ser produtora de toxinas termorresistentes, a sua presença nas plantas medicinais é preocupante (ROCHA, 2010). O S. aureus, embora resistente a condições mais baixas de Atividade de Água $(0,83)$ presentes nas amostras de boldo-do-chile, necessita estar presente em contagens iguais ou superiores a $106 \mathrm{UFC/g}$ para representar risco à humanos. Nestes níveis, o acúmulo de toxinas termorresistentes no material pode resultar em intoxicação nos consumidores que os utilize (FRANCO \& LANDGRAF, 2008; SILVA et al., 2007). Conforme exposto na tabela 1 , em todos os casos as populações de $S$. aureus observadas eram inferiores ao limite citado.

Os bolores e leveduras estavam presentes em $100 \%$ das amostras artesanais e $67 \%$ das industrializadas. Entretanto, em todas as amostras contidas neste percentual foram percebidas contagens superiores às verificadas no material proveniente da feira livre. A observação pode apontar a existência de falhas no processo de fabricação dos sachês, incluindo-se as etapas relacionadas à secagem e trituração das folhas. Adicionalmente, as amostras F(P2) e G (P3), apresentaram contagens de bolores e leveduras superiores ao nível recomendado pela OMS para materiais de origem vegetal destinado ao preparo de chás (WORLD HEALTH ORGANIZATION, 2007). 
Os microrganismos aeróbios mesófilos foram encontradas em todas as dez amostras analisadas. Seguindo o observado no caso dos bolores e leveduras, foram encontradas contagens superiores de aeróbios mesófilos em amostras industrializadas, evidenciando que ou o processo de beneficiamento industrial apresenta falhas, ou a estocagem do produto não é adequada.

\section{CONSIDERAÇÕES FINAIS}

A detecção de E. coli em $10 \%$ das amostras, de S. aureus em $50 \%$ destas, de bolores e leveduras, em $80 \%$ e de aeróbios mesófilos, em $100 \%$, demonstra que o material analisado apresenta baixa qualidade sanitária. Com base nas orientações de qualidade da OMS, duas das amostras industrializadas excederam os limites recomendados de bolores e leveduras para materiais de origem vegetal destinado ao preparo de chás e infusões (104 UFC/g), forma de uso predominante para o P. boldus Molina.

\section{REFERÊNCIAS}

BRASIL. Ministério das Minas e Energia. Projeto de fontes de abastecimento por água subterrânea no Estado do Rio Grande do Norte: Diagnóstico do Município de Currais Novos. Brasília, DF, 2005.

BUGNO, Adriana; ALMODOVAR, Adriana A. B.; PEREIRA, Tatiana C.; PINTO, T. de Jesus A.; SABINO, Myrna. Occurrence of toxigenic fungi in herbal drugs. Brazilian Journal of Microbiology, v. 37, p. 47-51, 2006.

FRANCO, B. D. G. M.; LANDGRAF, M. F. Microbiologia dos Alimentos. São Paulo: Atheneu, 2008.

GIL, Antônio Carlos. Como elaborar projetos de pesquisa. São Paulo: Atlas S. A., 1991.

GOOGLE. GOOGLE Earth. V. 6.2. Disponível em: http://www.google.com/intl/pt-PT/earth/index. Acesso em 21 jun. 2012.

MACIEL, M.A.M.; PINTO, A.C.; VEIGA JÚNIOR, V.F. Plantas medicinais- a necessidade de estudos multidisciplinares. Quim. Nova, v. 25, n. 3, 429-438, 2002.

NUNES, G.P.; SILVA, M.F. da; RESENDE, U.M.; SIQUEIRA, J.M. de. Plantas medicinais comercializadas por raizeiros do Centro de Campo Grande, Mato Grosso do Sul. Revista Brasileira de Farmacognosia, v. 13, n. 2, 83-92, 2003.

ROCHA, Francisco Angelo Gurgel da; MEDEIROS, Fábio Gonçalves Macêdo de. Contaminação microbiológica em amostras de Bauhinia forticata Link. comercializadas no município de Currais Novos, RN. In: IV Congresso de Pesquisa e Inovação da Rede Norte-Nordeste de Educação Tecnológica, Belém PA. IV CONNEPI: IFPA, 2009.

ROCHA, Francisco Angelo Gurgel da; MEDEIROS, Fábio Gonçalves Macêdo de; SILVA, Jonas Luiz Almada da; CHAGAS, Adailma de Brito. Microrganismos potencialmente produtores de toxinas em plantas medicinais. In: II Simpósio em Ciência e Tecnologia de Alimentos, 2010, Aracajú - SE. Avanços em Tecnologia de Alimentos, 2010.

SILVA, N. da; JUNQUEIRA, Valéria C. A.; SILVEIRA, N. F. A.; TANIWAKI, M. H.; SANTOS, R. F. S.; GOMES, R. A. R. Manual de métodos de análise microbiológica de alimentos. 3. ed. São Paulo: Varela, 2007.

VULCANO, Irma Regina Carrara; SILVEIRA, Josianne Nicácio; ALVAREZ-LEITE, Edna Maria. Teores de chumbo e cádmio em chás comercializados na região metropolitana de Belo Horizonte. Revista Brasileira de Ciências Farmacêuticas. v. 44. n.3. set, 2008.

WORLD HEALTH ORGANIZATION. Regulatory situation of herbal medicines: a worldwide review. Geneva, 1998.

WORLD HEALTH ORGANIZATION .WHO guidelines for assessing quality of herbal medicines with reference to contaminants and residues. Geneva: WHO Press, 2007. 